\title{
Suspected Adverse Effects After Human Papillomavirus Vaccination: A Temporal Relationship Between Vaccine Administration and the Appearance of Symptoms in Japan
}

\author{
Kazuki Ozawa $^{1}$ Akiyo Hineno ${ }^{1,2} \cdot$ Tomomi Kinoshita $^{1} \cdot$ Sakiko Ishihara $^{3}$. \\ Shu-ichi Ikeda ${ }^{2}$
}

Published online: 25 July 2017

(c) The Author(s) 2017. This article is an open access publication

\begin{abstract}
Introduction In Japan, after receiving human papillomavirus vaccination, a significant number of adolescent girls experienced various symptoms, the vast majority of which have been ascribed to chronic regional pain syndrome, orthostatic intolerance, and/or cognitive dysfunction. However, a causal link has not been established between human papillomavirus vaccination and the development of these symptoms.

Objective The aim of this study was to clarify the temporal relationship between human papillomavirus vaccination and the appearance of post-vaccination symptoms.
\end{abstract}

Kazuki Ozawa and Akiyo Hineno authors have contributed equally to this study.

Electronic supplementary material The online version of this article (doi:10.1007/s40264-017-0574-6) contains supplementary material, which is available to authorized users.

Shu-ichi Ikeda

ikedasi@shinshu-u.ac.jp

1 Department of Medicine (Neurology and Rheumatology), Shinshu University School of Medicine, Matsumoto, Japan

2 Intractable Disease Care Center, Shinshu University Hospital, Matsumoto 390-0802, Japan

3 Department of Rehabilitation, Shinshu University Hospital, Matsumoto, Japan
Methods Between June 2013 and December 2016, we examined symptoms and objective findings in 163 female patients who had received human papillomavirus vaccination. We used newly defined diagnostic criteria for accurate inclusion of patients who experienced adverse symptoms after human papillomavirus vaccination; these diagnostic criteria were created for this study, and thus their validity and reliability have not been established.

Results Overall, 43 female patients were excluded. Among the remaining 120 patients, 30 were diagnosed as having definite vaccine-related symptoms, and 42 were diagnosed as probable. Among these 72 patients, the age at initial vaccination ranged from 11 to 19 years (average $13.6 \pm 1.6$ years), and the age at appearance of symptoms ranged from 12 to 20 years (average $14.4 \pm 1.7$ years). The patients received the initial human papillomavirus vaccine injection between May 2010 and April 2013. The first affected girl developed symptoms in October 2010, and the last two affected girls developed symptoms in October 2015. The time to onset after the first vaccine dose ranged from 1 to 1532 days (average $319.7 \pm 349.3$ days). Conclusions The period of human papillomavirus vaccination considerably overlapped with that of unique postvaccination symptom development. Based on these sequential events, it is suggested that human papillomavirus vaccination is related to the transiently high prevalence of the previously mentioned symptoms including chronic regional pain syndrome and autonomic and cognitive dysfunctions in the vaccinated patients. 


\section{Key Points}

Using our new diagnostic criteria for adverse symptoms after human papillomavirus vaccination, 163 patients with various post-vaccination symptoms were examined and 72 were diagnosed with human papillomavirus vaccine-related symptoms.

The main symptoms consisted of chronic regional pain syndrome, and autonomic and cognitive dysfunctions. The most frequent objective findings were orthostatic dysregulation, flattened digital plethysmography, and focal abnormality of cerebral blood flow in single-photon emission computed tomography.

The time to onset after the first vaccine dose showed a wide range (average $319.7 \pm 349.3$ days).

The period of human papillomavirus vaccination in Japan considerably overlapped with that of the development of unique symptoms in the vaccinated patients.

\section{Introduction}

Human papillomavirus (HPV) vaccines have been introduced worldwide for the prevention of uterine and cervical cancer [1, 2]. In May 2010, Cervarix ${ }^{\circledR}$ (GlaxoSmithKline, Research Triangle Park, NC, USA), a papillomavirus recombinant bivalent vaccine, and Gardasil ${ }^{\circledR}$ (Merck \& Co, Inc., Whitehouse Station, NJ, USA), a papillomavirus recombinant quadrivalent vaccine, were widely introduced for use in female Japanese teenagers, as the cost of this vaccination was largely borne by several local governments. From April 2013, female adolescents aged 13-16 years were legally required to undergo this vaccination, and its cost was fully covered by the government [3]. Soon after this law went into effect, a high incidence of chronic limb pain and weakness, frequently complicated by violent tremulous involuntary movement, was noted in Japanese girls after HPV vaccination. According to the reports of a special committee organized in the Japanese Ministry of Public Health, Labour and Welfare [4, 5], by the end of March 2013, 8,646,147 doses of HPV vaccines had been used in Japan; it was further estimated that 3.28 million girls received this vaccine. Based on medical institution reports, 1196 cases were reported to have adverse reactions, of which 106 cases were considered to be serious. These symptoms were largely reported by the Japanese mass media as possible adverse events of HPV vaccination. Additionally, a social community was organized in Tokyo (All Japan Coordinating Association of HPV Vaccine Sufferers) [6], and this voluntary community started to collect more information from all over Japan. At the end of June 2013, the Japanese Ministry of Public Health, Labour and Welfare, withdrew the recommendation for use of this vaccination [7], and our institution was asked by the ministry to examine these affected adolescent Japanese girls.

The number of adverse events after HPV vaccination has been increasing, although the use of this vaccine has dramatically decreased during the recent 3.5 years. According to the latest reports of a Japanese special committee [8, 9], by the end of November 2016, a total of $8,931,261$ doses of HPV vaccines were administered to about 3.39 million girls in Japan; 2024 cases were reported to experience adverse reactions, of which 673 were considered to have serious symptoms. During this term, the increase in the number of vaccinated girls was about 0.11 million, while that of case reports with serious adverse reactions was 567. The affected vaccine recipients reported various symptoms such as intractable headache, long-lasting general fatigue, widespread pain involving the limbs, chest, and abdominal wall, and decreased learning ability; most of these symptoms were ascribed to orthostatic intolerance, chronic regional pain syndrome (CRPS) [10], and/or cognitive dysfunction [11]. Moreover, clinical manifestations of suspected vaccine-related adverse effects after HPV vaccination have been reported to be ascribed to abnormal autoimmune reactions [12, 13], but the affected patients do not present with any characteristic laboratory findings, including a lack of anti-neural autoantibodies in serum or cerebrospinal fluid [10].

Demonstrating a causal link between HPV vaccination and the development of these symptoms is a very challenging task, particularly because there seem to be few tools or applicable algorithms to demonstrate direct causation. Therefore, in this study, we attempted to clarify the clinical spectrum of HPV vaccine-related symptoms, and then investigated the temporal relationship between HPV vaccination and the appearance of these unique symptoms, which may provide valuable insight into the pathogenesis of this remarkable phenomenon that occurred after HPV vaccination.

\section{Patients and Methods}

Between June 2013 and December 2016, a total of 163 consecutive female patients visited our institution complaining of multiple symptoms after HPV vaccination. Among them, 114 had undergone vaccination with Cervarix $^{\circledR}$ and 43 with Gardasil ${ }^{\circledR}$. No accurate information was 
available on the type of vaccine administered to the remaining six patients. Using self-reported answers to printed questionnaires (Electronic Supplementary Material) for our institute and medical records from other institutes when available, we obtained a detailed history of the patients' symptoms to determine the duration between vaccination and the development of the first symptom(s) suspected to be related to the vaccine. The patients underwent complete physical and neurological examinations and routine laboratory screening, including measurement of the serum levels of C-reactive protein.

Patients with hypotension and/or coldness of the limbs were evaluated with the Schellong test and a digital plethysmogram. In the Schellong test, orthostatic hypotension was defined as a decrease of more than $20 \mathrm{mmHg}$ in systolic blood pressure and/or $5 \mathrm{mmHg}$ in diastolic pressure within a few minutes after standing [14]. Postural orthostatic tachycardia syndrome (POTS) was defined as an increase in heart rate of more than 30 beats per minute $(\mathrm{bpm})$ within the first $10 \mathrm{~min}$ of standing or tilting the head upwards [15]. A digital plethysmogram was recorded from the right second finger and right first toe, while checking the patients' skin temperature. The digital plethysmogram findings were evaluated according to the classification of the wave pattern [16]. The presence of a peripheral plateau pattern showing a markedly reduced height of the waves, with loss of a notch in the latter half of the wave, was judged to be pathological [10]. No standard diagnostic criterion exists for diminished skin temperature; therefore, a value below room temperature was considered abnormal.

Neuropsychological tests and functional brain imaging were performed in patients who complained of decreased learning ability and/or memory loss, and those without a history of any psychiatric or anxiety disorders. The neuropsychological tests used were the Wechsler Adult Intelligence Scale-Third Edition (WAIS-III) and the Trail Making Test (TMT). The WAIS-III scores were evaluated using the following intelligence quotient (IQ) scales: full scale IQ, verbal IQ, performance IQ, verbal comprehension IQ, working memory IQ, perceptual organization IQ, and processing speed. A score below 80 on each scale of the WAIS-III was determined to be abnormal. In the TMT, the time taken by study patients to complete part B was compared with the time taken by age-matched controls [17], and significant prolongation of the time taken to complete part B (>95.0 s average time \pm 2 standard deviations) was defined as abnormal. Finally, patients with abnormal results in either the WAIS-III or the TMT were considered to have impaired cognitive function. Functional brain imaging was performed using single-photon emission computed tomography (SPECT). Single-photon emission computed tomography was performed using a two-head rotating gamma camera (Symba T6; Siemens, Berlin, Germany) equipped with parallel hole collimators: these images were acquired 15 min after intravenous injection of body weight $(\mathrm{kg}) \times 2.5-3.5 \mathrm{MBq} N$-isopropyl- ${ }^{123} \mathrm{I}-p$ iodoamphetamine. The data obtained from ${ }^{123} \mathrm{I}-p$-iodoamphetamine SPECT were analyzed using the three-dimensional stereotactic surface projection technique to identify brain regions with abnormal perfusion. Focal hypoperfusion observed in any of the cerebral cortices except the occipital cortex was considered abnormal. Data are presented as mean values and standard deviations.

\section{Results}

For accurate selection of patients affected by suspected vaccine-related symptoms, we first prepared new diagnostic criteria for vaccine-related adverse events following HPV vaccination (Table 1) based on our previous report [10] and the inclusion and exclusion criteria for vaccinerelated adverse events following HPV vaccination proposed by Nishioka et al. [18] and Matsudaira et al. [11]. These diagnostic criteria were created for this study, and thus their validity and reliability have not been established. Between June 2013 and December 2016, 163 female patients were examined (Fig. 1). Of these, we excluded 15 who displayed symptoms before vaccination and four who received HPV vaccine after the age of 30 years. An additional 24 patients whose symptoms or disorders were explainable by known diseases or who had abnormal results in the laboratory blood tests we conducted were also excluded: six with epilepsy, five with psychiatric or anxiety disorders, three with systemic lupus erythematosus, and two with minor abnormality in routine laboratory data. Concerning the last two excluded patients, one 17-year-old patient, who received HPV vaccination at the age of 14 years, was referred to us because of migrating joint pain, limb shaking, and a slightly elevated C-reactive protein level $(0.74 \mathrm{mg} / \mathrm{dL}$, normal $<0.10 \mathrm{mg} / \mathrm{dL})$. She showed coldness in the feet but lacked either swelling or redness of joints and thus, her clinical features indicated HPV vaccine-related adverse effects. However, a close assessment of her laboratory data suggested juvenile idiopathic arthritis as the possible cause of her symptoms, not from adverse effects after HPV vaccination. The other patient aged 17 years had been experiencing general fatigue, headache, lower limb pain, and intermittent high fever after HPV vaccination, and showed cervical and axillar lymphadenopathy with an elevated serum C-reactive protein level $(1.40 \mathrm{mg} / \mathrm{dL})$. She seemed to have HPV vaccinerelated adverse effects including reactive lymphadenopathy. However, histiocytic necrotizing lymphadenitis (Kikuchi disease), which is occasionally seen in Asian 
Table 1 Diagnostic criteria for suspected adverse effects after human papilloma virus (HPV) vaccination

\section{Prerequisite}

1. At least one exposure to HPV vaccination

2. No abnormality of a physical or psychological condition before HPV vaccination

3. Appearance of clinical manifestations after HPV vaccination

II. Major symptoms

1. Prolonged general fatigue (lasting for more than 4 weeks)

2. Chronic headache, especially after standing up

3. Widespread pain (migratory joint pain, limb pain, or myalgia)

4. Limb shaking (tremor or myoclonus like)

5. Dysautonomic symptoms (orthostatic fainting, postural orthostatic tachycardia, or delayed or rapid gastrointestinal motility)

6. Motor dysfunction (frequent sudden falls, limb weakness or paralysis, gait disturbance)

7. Abnormal sensation (coldness in limbs, limb paresthesia, photophobia)

8. Sleep disturbance (hypersomnia, insomnia)

9. Learning impairment (memory impairment, difficulties in concentration, verbal dyspraxia)

10. Menstrual abnormality (amenorrhea, hypermenorrhea, irregular menstruation)

III. Objective findings

1. Persistent hypotension

2. Orthostatic hypotension or postural tachycardia on standing or tilt table test (Schellong test)

3. Decreased skin temperature

4. Peripheral plateau pattern in digital plethysmogram

5. Decreased cognitive function test

6. Decreased regional cerebral blood flow as shown in brain single-photon emission computerized tomography

IV. Exclusion

1. Abnormality in routine blood laboratory data

2. Conditions compatible with diagnostic criteria of other diseases (e.g., juvenile idiopathic arthritis, epilepsy, and autism spectrum disorder)

3. HPV vaccination after 30 years of age

Definite case: more than five of ten major symptoms + more than three of six objective findings + no exclusive conditions

Probable case: more than five of ten major symptoms + no exclusive conditions

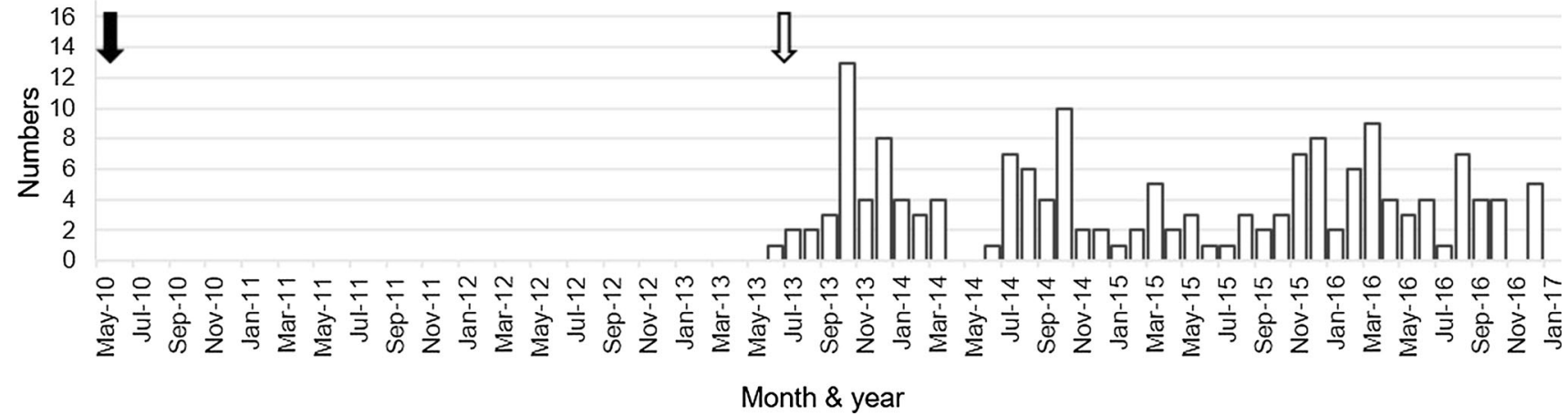

Fig. 1 Number of patients who were suspected to have adverse symptoms after human papillomavirus vaccination and then visited our institution in each month: 31 patients in 2013, 45 in 2014, 38 in 2015, and 49 in 2016, totaling 163 female patients. The black arrow

adolescent girls, could not be ruled out. Thus, to avoid possible biases, we applied strict exclusion criteria including any minor abnormality in routine laboratory data on this study.

The clinical manifestations and objective findings for the remaining 120 patients were analyzed according to our indicates the active start of human papillomavirus vaccination in Japan and the white arrow indicates the recommendation to stop this vaccination

new diagnostic criteria (Table 1): 30 patients were diagnosed as having definite vaccine-related symptoms, and 42 were diagnosed as having probable vaccine-related symptoms. The summary of symptoms and signs of diagnosed (definite and probable) and undiagnosed patients are shown in Table 2, and objective findings for these patients are 


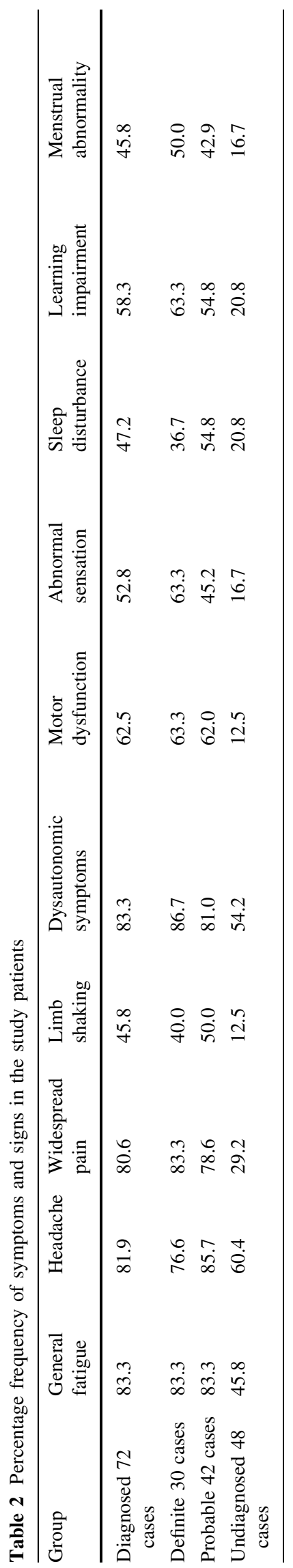

presented in Table 3 . The distribution of patients by age at the initial vaccination and at the appearance of symptoms is shown in Fig. 2. Among the 72 girls diagnosed as having definite or probable vaccine-related symptoms, the age at the initial vaccination ranged from 11 to 19 years (average $13.6 \pm 1.6$ years; Fig. 2a), the age at the appearance of symptoms ranged from 12 to 20 years (average $14.4 \pm 1.7$ years; Fig. 2b), and the time to onset from the first vaccine dose ranged from 1 to 1532 days (average $319.7 \pm$ 349.3 days). The appearance of symptoms was reported after the first vaccination in $16.7 \%$ of the affected girls, after the second vaccination in $29.2 \%$, after the third vaccination in $52.8 \%$, and unknown in $1.3 \%$. The interval between the onset of symptoms and our initial examination ranged from 0 to 63 months (average duration $28.0 \pm$ 15.7 months) and this interval indicated the duration of illness in the patients until they visited us.

Symptoms or signs frequently observed in these 72 girls were prolonged general fatigue, chronic headache, widespread pain, limb shaking, dysautonomic symptoms, motor dysfunction, abnormal sensation, sleep disturbance, learning impairment, and menstrual abnormality (Table 2). The affected girls usually stayed in bed until noon because of marked general fatigue, possibly combined with sleep disturbance, and upon awakening, they experienced a continuous headache of varying severity. Dysautonomic symptoms included frequent squatting or syncope during their daily activities. Bowel dysfunctions manifested as frequent abdominal pain with diarrhea or chronic constipation with the abdominal distension. They also experienced widespread pain involving the limbs and trunk. In the limbs, migratory joint pain with no signs of inflammation was typical, whereas in the trunk, intermittent neuralgic pain in the chest wall or abdominal wall was common, and back pain or lumbago was sometimes observed. Motor dysfunction showed variable patterns: distal dominant weakness of the limbs, mimicking the symptoms of polyneuropathy, hemiplegia closely resembling that observed after stroke, and paraplegia or tetraparesis. In these conditions, demonstrable classical neurological signs, such as areflexia, exaggerated deep tendon reflexes, or Babinski sign, were absent. Abnormal sensations were mainly reported in the thigh or lower legs: dysesthesia or allodynia was common, and shooting or burning pain and complaints such as restless leg syndrome were occasionally reported. However, we did not detect any objective sensory impairment upon pin-prick or vibratory stimulations. Regarding learning impairment, the affected girls claimed that they could not remember long sentences from their textbooks or understand different themes simultaneously.

Objective findings that occurred at a high frequency were orthostatic dysregulation, abnormal digital 
Table 3 Percentage frequency (positive cases/total cases examined) of abnormal objective findings in the patients examined

\begin{tabular}{|c|c|c|c|c|c|c|c|}
\hline \multirow[t]{2}{*}{ Group } & \multirow[t]{2}{*}{ Hypotension } & \multirow[t]{2}{*}{$\begin{array}{l}\text { Orthostatic } \\
\text { intolerance }\end{array}$} & \multirow[t]{2}{*}{$\begin{array}{l}\text { Decreased } \\
\text { skin } \\
\text { temperature }\end{array}$} & \multirow[t]{2}{*}{$\begin{array}{l}\text { Flattened digital } \\
\text { plethysmography }\end{array}$} & \multicolumn{2}{|c|}{$\begin{array}{l}\text { Abnormality in } \\
\text { cognitive } \\
\text { function test }\end{array}$} & \multirow[t]{2}{*}{$\begin{array}{l}\text { Focal abnormality } \\
\text { of CBF in SPECT }\end{array}$} \\
\hline & & & & & $\begin{array}{l}\text { WAIS- } \\
\text { III }\end{array}$ & TMT & \\
\hline $\begin{array}{r}\text { Diagnosed } 72 \text { cases (positive } \\
\text { cases/total cases examined) }\end{array}$ & $41.7(30 / 72)$ & $\begin{array}{l}70.8(34 / 48) \\
\text { (OH 17, POTS } \\
17)\end{array}$ & $37.5(21 / 56)$ & $70.2(40 / 57)$ & $\begin{array}{c}47.6 \\
(10 / \\
21)\end{array}$ & $\begin{array}{r}43.8 \\
(7 / \\
16)\end{array}$ & $75.0(12 / 16)$ \\
\hline $\begin{array}{l}\text { Definite } 30 \text { cases (positive } \\
\text { cases/total cases examined) }\end{array}$ & $46.7(14 / 30)$ & $\begin{array}{l}83.3(25 / 30) \\
\text { (OH 12, POTS } \\
13)\end{array}$ & $62.1(18 / 29)$ & $86.2(25 / 29)$ & $\begin{array}{r}58.3 \\
(7 / \\
12)\end{array}$ & $\begin{array}{r}50.0 \\
(5 / \\
10)\end{array}$ & $88.9(8 / 9)$ \\
\hline $\begin{array}{l}\text { Probable } 42 \text { cases (positive } \\
\text { cases/total cases examined) }\end{array}$ & $38.1(16 / 42)$ & $\begin{array}{l}50.0(9 / 18)(\mathrm{OH} \\
5, \text { POTS } 4)\end{array}$ & $11.1(3 / 27)$ & $53.6(15 / 28)$ & $\begin{array}{l}33.3 \\
(3 / 9)\end{array}$ & $\begin{array}{l}33.3 \\
(2 / \\
6)\end{array}$ & $57.1(4 / 7)$ \\
\hline $\begin{array}{l}\text { Undiagnosed } 48 \text { cases (positive } \\
\text { cases/total cases examined) }\end{array}$ & $29.2(14 / 48)$ & $\begin{array}{l}50.0(8 / 16)(\mathrm{OH} \\
6, \text { POTS 2) }\end{array}$ & $28.0(7 / 25)$ & $36.0(9 / 25)$ & $\begin{array}{l}25.0 \\
(2 / 8)\end{array}$ & $\begin{array}{c}14.3 \\
(1 / \\
7)\end{array}$ & $50.0(3 / 6)$ \\
\hline
\end{tabular}

$C B F$ cerebral blood flow, $O H$ orthostatic hypotension, POTS postural orthostatic tachycardia syndrome, SPECT single-photon emission computerized tomography, TMT Trail Making Test, WAIS-III Wechsler Intelligence Scale-Third Edition

Fig. 2 Age distribution of patients diagnosed as having human papillomavirus vaccinerelated symptoms. a Age distribution at an initial human papillomavirus vaccination. b Age at the development of symptoms. Black bar definite cases, gray bar probable cases
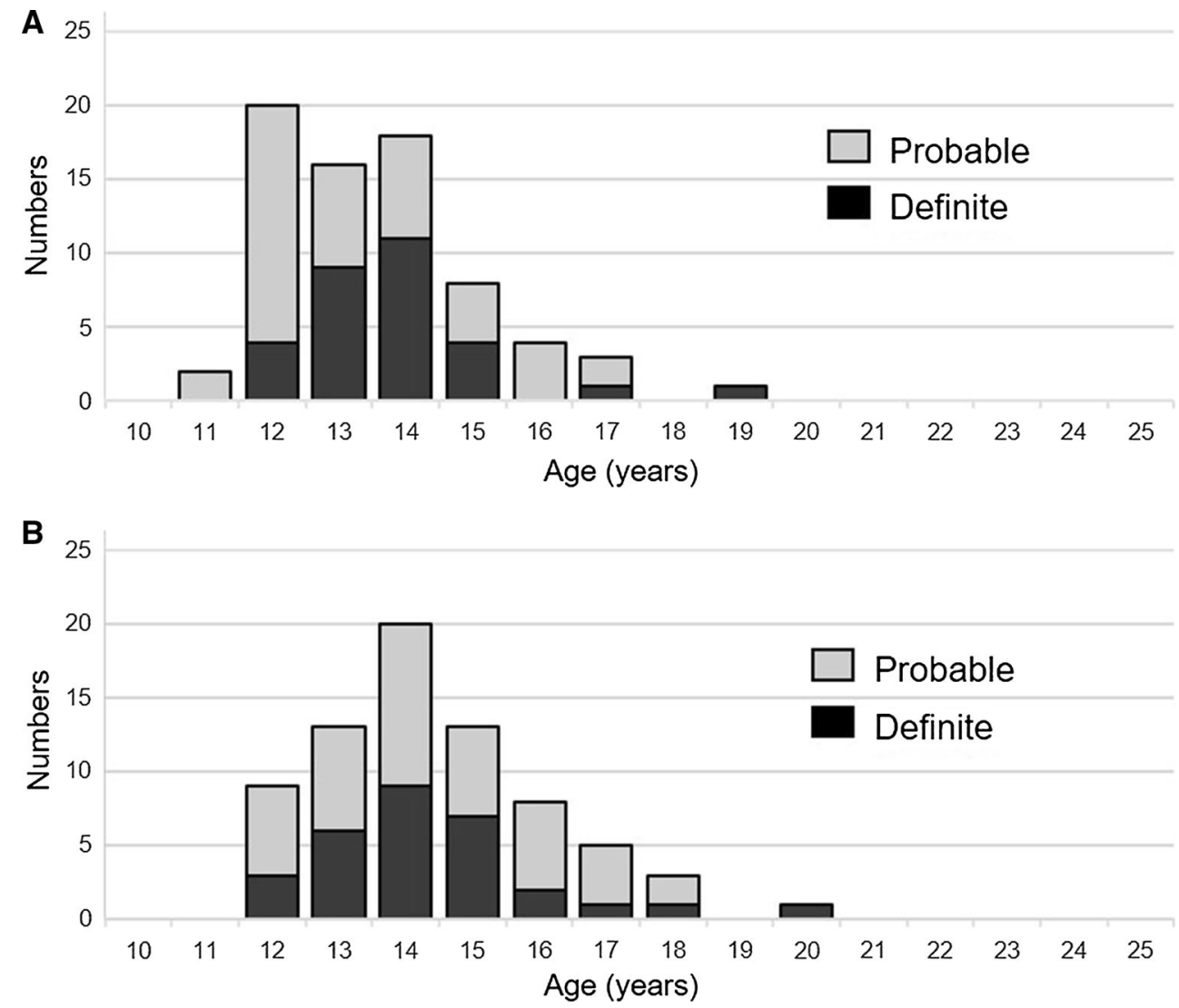

plethysmogram recordings, and abnormalities on SPECT images (Table 3); POTS was detected in half of the girls with orthostatic dysregulation. In the SPECT images, focal hypoperfusion areas, which were located mainly in the medial fronto-parietal and temporo-parietal regions, were found in 12 of the 16 diagnosed patients who underwent nuclear scanning of the brain. Corresponding with the findings of SPECT images, decreased scores on cognitive function tests were seen: although the WAIS-III scores showed no consistent abnormality in the patients examined, a relatively high frequency of decreased scores was observed in performance IQ (6 of 21 girls; 28.6\%), 

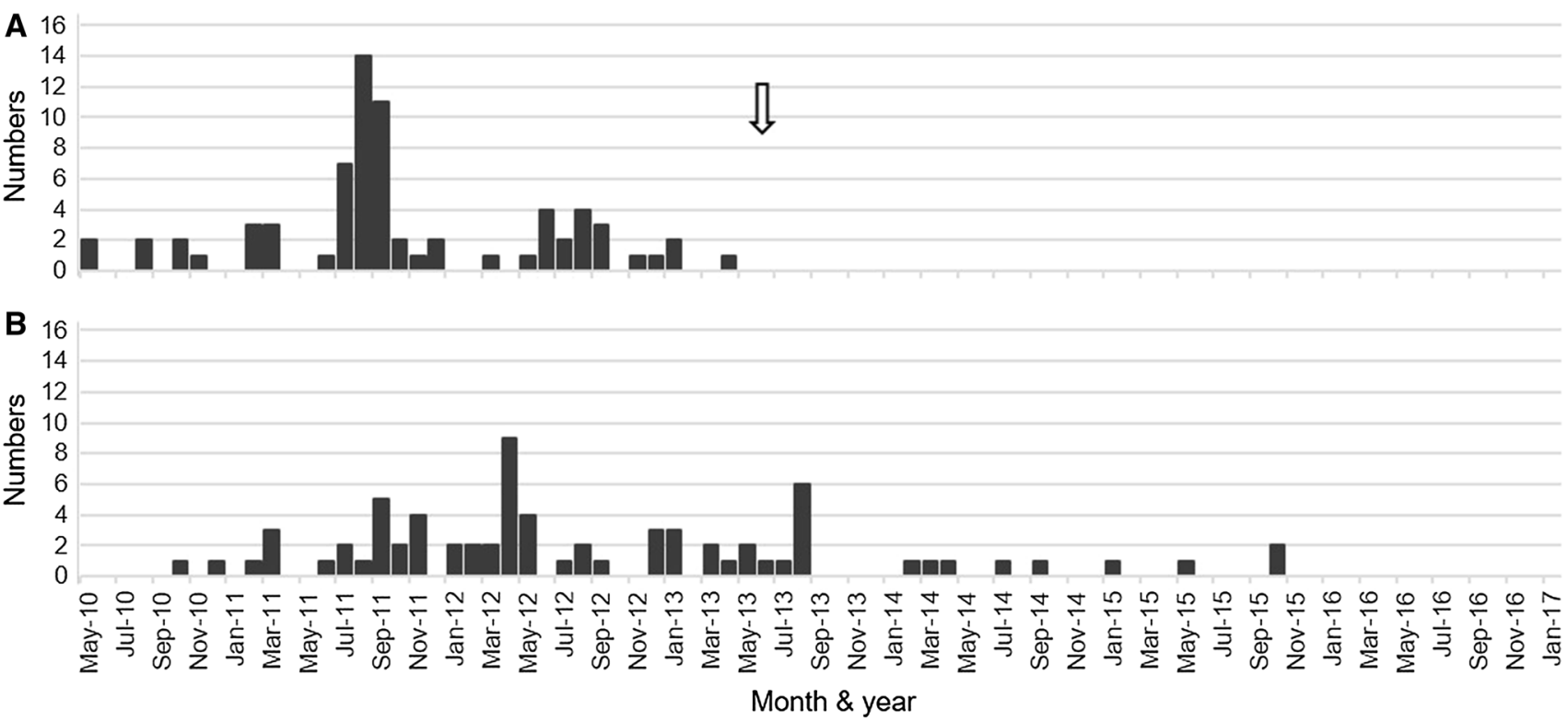

Fig. 3 Temporal relationship between human papillomavirus (HPV) vaccination and the development of symptoms in patients diagnosed as having HPV vaccine-related symptoms. The period ranges from May 2010 to December 2016. a Number of patients who received the first injection of HPV vaccine each month. The peak period seems to

perceptual organization IQ ( 5 of $21 ; 23.8 \%$ ), and processing speed test ( 5 of $21 ; 23.8 \%$ ). In the TMT, about half of the patients tested revealed prolonged times ( 7 of 16 ; $43.8 \%)$.

Regarding symptom severity of these 72 patients at our initial examination, 30 patients had symptoms, but they could independently perform their daily activities including schoolwork. In 38 patients, daily activities were significantly impaired owing to the presence of symptoms: they were not able to wake in the morning and needed help to leave home. Four patients were confined to a wheelchair, and spent most of the day in bed.

We also investigated the temporal relationship between vaccine administration and the appearance of symptoms in the 72 girls diagnosed as having definite or probable vaccine-related symptoms. The first of three HPV vaccination injections started in May 2010 and was terminated in April 2013 (Fig. 3a). The first affected girl developed symptoms in October 2010, and the latest appearance of symptoms occurred in two girls in October 2015 (Fig. 3b). Over the previous 14 months, we have not examined any female patients who were freshly affected by these unique symptoms (Fig. 3b).

\subsection{Representative Case Report}

In November 2014, a 17-year-old girl visited our institution because of limb pain, memory loss, and decreased calculation ability. She had received three injections of HPV be between July 2011 and September 2012. An arrow indicates the time when the Japanese Ministry of Public Health, Labour and Welfare stopped recommending HPV vaccination. b Number of patients who developed symptoms each month. The peak period appears to be between September 2011 and August 2013

vaccine by the end of July 2011. Two months after the third vaccination, she experienced pain and unpleasant coldness bilaterally in her shoulders and forearms, sometimes resulting in difficulty in writing. This symptom lasted for 6 months, followed by spontaneous relief. She subsequently experienced marked general fatigue, bowel dysfunction, tachycardia, hyperventilation, and irregular menstruation. Since April 2013, a marked increase in the number of episodes of severe headaches, especially in the occipital area, was noted. During these headaches, she experienced short-term asthenia with aphonia, and after repeated attacks, she experienced transient memory disturbance, including the inability to remember her address and name, difficulty in concentrating while studying, and sleep disorder. She underwent computed tomography, magnetic resonance imaging, and routine blood examinations at other hospitals, all of which revealed no abnormalities. Her symptoms resulted in frequent absenteeism at school in 2014.

Our physical examination revealed that the patient was $159.0 \mathrm{~cm}$ tall and weighed $46.1 \mathrm{~kg}$. Her body temperature was $36.8^{\circ} \mathrm{C}$. Although she appeared to be in an asthenic state, a general physical examination and neurological examination showed no abnormality. Results of routine laboratory tests were all normal. In the Schellong test, her pulse rate was $90 \mathrm{bpm}$, with a blood pressure of $108 / 65 \mathrm{mmHg}$ in the sitting position. 2 min after standing up, her pulse rate increased to $129 \mathrm{bpm}$, without a significant change of blood pressure, and this tachycardia 


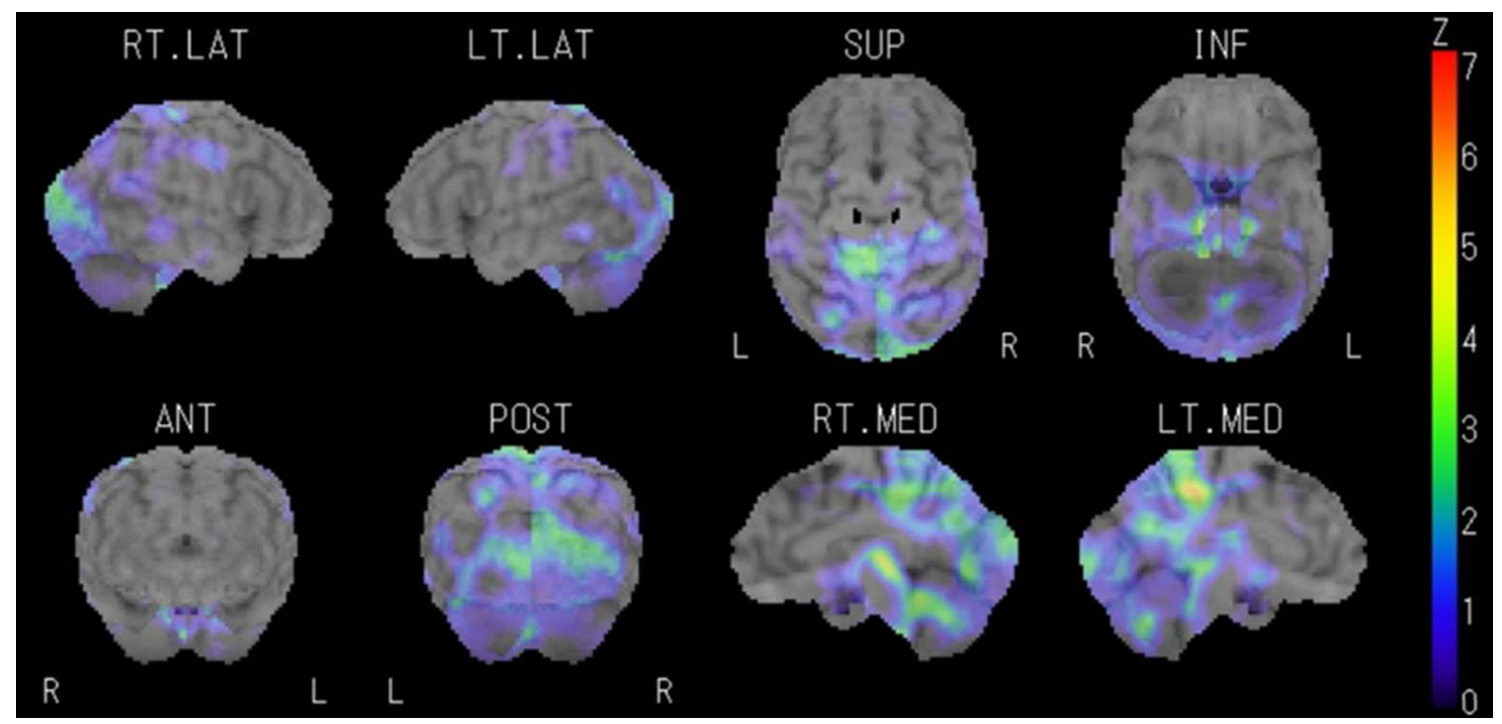

Fig. 4 Three-dimensional stereotactic, surface projection, singlephoton emission computerized tomography images from the representative case presented. Areas with focal hypoperfusion are visible

continued for more than $10 \mathrm{~min}$, causing her to squat and resulting in the termination of the examination. Her skin temperature was $24.8^{\circ} \mathrm{C}$ in the right second finger and $24.0^{\circ} \mathrm{C}$ in the right first toe, at a room temperature of $26.5^{\circ} \mathrm{C}$. Plethysmograms of the finger and toe showed markedly reduced wave heights. Based on her medical history and our objective findings, we concluded that she had POTS and disturbed sympathetic innervation on skin, and we performed further examinations.

Her WAIS-III scores were normal: full scale IQ 107, verbal IQ 115, performance IQ 95, verbal comprehension 111, working memory 92, perceptual organization 95, processing speed 84 . In contrast, the time taken to complete part B of the TMT was prolonged (110.46 s; controls $<95.0$ $\mathrm{s}$ ), indicating impaired frontal lobe function. SPECT showed focal hypoperfusion in both medial parieto-temporal cortices (Fig. 4). She was diagnosed as having cognitive dysfunction and was finally classified as a definite case because of the presence of nine major symptoms and five objective findings.

\section{Discussion}

Vaccinations are generally safe and are expected to significantly reduce morbidity and mortality in the targeted infections, but they occasionally result in some adverse effects. For example, unexpected adverse effects following HPV vaccination were reported to the Japanese Ministry of Public Health, Labour and Welfare during 3 months after the national vaccination program, including HPV vaccination, started [3], and the mandatory vaccination with on the medial sides of the parieto-temporal cortices. $R T R$ right, $L T$ $L$ left, $L A T$ lateral, SUP superior, $I N F$ inferior, $A N T$ anterior, $P O S T$ posterior, $M E D$ medial, $Z Z$ score

HPV vaccine was terminated at the end of June 2013 [7]. However, a causal relationship between HPV vaccination and various post-vaccination symptoms is controversial in Japan [19], and many of the affected girls were initially diagnosed as having psychiatric illness because of the absence of pathologic radiological images and specific abnormalities in laboratory test results. Thus, in this study, we first proposed new diagnostic criteria for adverse symptoms after HPV vaccination. Subsequently, using these criteria, 163 patients with various post-vaccination symptoms were evaluated and 72 were diagnosed with HPV vaccine-related symptoms. On the basis of these 72 the clinical spectrum of HPV vaccine-related symptoms was clarified, adding the frequent objective findings of orthostatic dysregulation, flattened digital plethysmography, and focal abnormality of cerebral blood flow on SPECT. Finally, we investigated the temporal relationship between HPV vaccination and the appearance of these unique symptoms for the purpose of addressing causality, and have revealed that the period of HPV vaccination in Japan considerably overlapped with that of the development of unique symptoms in the vaccinated patients.

The Vaccine Adverse Event Reporting System is available in USA to monitor the incidence of vaccine-related adverse events. Analysis of 12,424 Vaccine Adverse Event Reporting System reports following HPV vaccination revealed that syncope, dizziness, and headache were the most prevalent post-vaccination events [20]. The suspected HPV vaccine-related adverse events observed in this study are largely different from those mentioned above; estimating from the interval between the onset of symptoms and patients' visits to us, adverse events lasted 
for a long time and were severe enough to affect the patients' quality of life negatively. The female patients examined in this study presented with similar histories and pattern of clinical manifestations as the participants in our previous study: in our previous report that included 40 girls with possible HPV vaccine-related adverse effects, common symptoms were headache, general fatigue, limb pain, and weakness [10]. All of these symptoms could be explained by a combination of orthostatic dysregulation and CRPS [21]. In addition, peripheral sympathetic nerve dysfunction was responsible for the appearance of orthostatic dysregulation and CRPS [10, 22]. Recently, cognitive dysfunction has been noted as a possible adverse effect that manifests late after HPV vaccination [11]: it manifests mainly as memory impairment, decreased calculation ability, and transient prosopagnosia-like symptoms; affected patients experience difficulties in performing their schoolwork, with some being absent from school for long periods. Single-photon emission computed tomography imaging of the brains of these patients revealed a significant reduction in regional cerebral blood flow in functionally related cerebral gyri such as the right medial, frontal, and/or right rectal gyri [11]. Additionally, patients' conditions are more complicated with sleep disorders [23] and neuro-endocrine dysfunction [24]. Furthermore, abnormal immune responses were observed in cerebrospinal fluid samples collected from the patients with cognitive dysfunction [25].

Similar data were reported for patients from Denmark [26, 27], Italy [28], and the other countries [12, 29-31], and the pathogenesis of this post-vaccination syndrome has been postulated to be caused by an autoimmune disorder including autoimmune/inflammatory syndrome induced by adjuvants [12, 13, 32]. The clinical characteristics of autoimmune/inflammatory syndrome induced by adjuvants seem to be similar to that of possible HPV vaccine-related adverse events discussed here [33]. Additionally, causative autoantibodies for $\beta-2$ adrenergic and M2 muscarinic receptors were also detected in two female POTS patients with HPV vaccine-related adverse symptoms [34, 35].

In this series of the patients, it is notable that the time to onset from the first vaccine dose shows a wide range and the average of that is very long in comparison with the adverse effects of conventional vaccinations. Another Japanese research group reported that the time to onset from the first vaccine dose ranged from 1 day to 43 months with a mean period of $14.0 \pm 11.6$ months on the basis of the investigation of the post-vaccinated 35 girls [24]. Many symptoms after HPV vaccination are explainable by a combination of autonomic dysfunction, CRPS, and cognitive dysfunction, and all three disorders are characterized by insidious onset and progression. It is rather difficult to determine the exact time of onset in these symptoms. For example, some patients did not alter their lifestyle even with general fatigue and limb disorders, and only after repeated attacks of syncope did they recognize the possible adverse effects of HPV vaccination. Thus, the interval from an initial injection of HPV vaccine until the first awareness of these symptoms may vary among the involved patients.

In the analysis of the temporal relationship between HPV vaccination and the onset of symptoms, the peak onset of symptoms in the vaccinated girls occurred at an age similar to that in girls with HPV vaccination; the difference in average age between both groups was about 8 months, which is relatively similar to the average interval of 319.5 days between the first HPV vaccine administration and the onset of symptoms. Regarding the time course of HPV vaccination and symptom onset, most of the patients we examined received HPV vaccination between May 2010 and April 2013, the peak period being between July 2011 and September 2012. However, the development of symptoms after HPV vaccination in the same group of patients was between October 2010 and the same month 2015, the peak period being between September 2011 and August 2013; thus, all these four periods considerably overlapped, especially for the peak periods of both events. Therefore, these two events could be related to each other.

Among several iatrogenic disorders after injections, transmission of human immunodeficiency virus or hepatitis virus to individuals can be demonstrable after the appearance of virus-induced symptoms [36, 37]. Although there is no tool to obtain direct evidence of the role of HPV vaccination in the development of adverse symptoms in the present study, the time course of symptoms indicates that the manifestation of HPV vaccine-related symptoms terminated around October 2015, which was 28 months after the recommendation for HPV vaccination was withdrawn in Japan. However, POTS or CRPS occasionally develops in adolescent girls [14, 38], and a questionnaire-based study performed during July to December in 2015 reported that a group of symptoms including CRPS and POTS, which were seen in HPV-vaccinated girls, certainly occurred in Japanese girls with no history of HPV vaccination and its incidence was at least 5.3/100,000 [39].

This study has some limitations. We were unable to recruit a satisfactory control group from the general unvaccinated population. The next limitation is the small number of study subjects, all of whom were referred to us. The total number of Japanese girls with HPV vaccine-related symptoms would be much higher. Further large-scale studies are needed to elucidate the pathogenesis of this unusual phenomenon. To this end, a murine pathological model was recently established by combined administration of HPV vaccine and pertussis toxin [40], which might be helpful for investigating immune-related pathological conditions in this post-vaccinated disorder. 


\section{Conclusion}

The present study revealed that the age distribution patterns in female adolescent patients who received HPV vaccination and that in the female patients who experienced HPV vaccine-related symptoms are very similar. Moreover, the period of HPV vaccination in Japan overlapped with that of the development of HPV vaccine-related symptoms in the vaccinated patients. Moreover, 28 months have passed since the recommendation for HPV vaccination was withdrawn, and new HPV vaccine-related symptoms have not been observed during our 14-month follow-up period. The sequence of these events suggests that HPV vaccination is temporally related to the development of these symptoms in Japanese adolescent girls. Further large-scale studies are required to clarify the pathophysiology of these symptoms and to establish the necessary treatment for affected patients.

Acknowledgements The authors are most grateful to Dr. Shin Yanagisawa at the Department of Radiology in our hospital for conducting single-photon emission computed tomography examinations. The authors also thank Editage (http://www.editage.jp) for English language editing.

\section{Compliance with Ethical Standards}

Funding This study was supported by a grant from a Health and Labour Science Research Grant on Emerging and Re-emerging Infectious Diseases (Establishment of Treatments and Providing Information on Symptoms after HPV vaccination, H28-001) to S.I. from the Ministry of Public Health, Labour and Welfare, Japan.

Conflict of interest Kazuki Ozawa, Akiyo Hineno, Tomomi Kinoshita, Sakiko Ishihara, and Shu-ichi Ikeda have no conflicts of interest directly relevant to the content of this study.

Ethics approval The study protocol was carried out in accordance with the Institutional Review Board approval (Nos. 3621 and 3659) of Shinshu University School of Medicine, Nagano, Japan.

Consent to participate Written informed consent was obtained from the patient for publication of her case report. A copy of the written consent may be requested for review from the corresponding author.

Open Access This article is distributed under the terms of the Creative Commons Attribution-NonCommercial 4.0 International License (http://creativecommons.org/licenses/by-nc/4.0/), which permits any noncommercial use, distribution, and reproduction in any medium, provided you give appropriate credit to the original author(s) and the source, provide a link to the Creative Commons license, and indicate if changes were made.

\section{References}

1. FUTURE II study group. Quadrivalent vaccine against human papillomavirus to prevent high-grade cervical lesions. N Engl J Med. 2007;356:1915-27.
2. Jeurissen S, Makar A. Epidemiological and economic impact of human papillomavirus vaccines. Int $\mathrm{J}$ Gynecol Cancer. 2009;19:761-71.

3. The Ministry of Health, Labour and Welfare. http://www.mhlw. go.jp/topics/bcg/tp250330-2.html. 2017. Accessed 16 Jul 2017. (in Japanese).

4. The Ministry of Health, Labour and Welfare. http://www.mhlw. go.jp/stf/shingi/2r98520000034g8f-att/2r98520000034hr8_2.pdf. 2017. Accessed 16 Jul 2017. (in Japanese).

5. The Ministry of Health, Labour and Welfare. http://www.mhlw. go.jp/stf/shingi/2r98520000034g8f-att/2r98520000034hrr_2.pdf. 2017. Accessed 16 Jul 2017. (in Japanese).

6. All Japan Coordinating Association of HPV Vaccine Sufferers . http://hpvv-danger.jp (in Japanese). 2017. Accessed 16 Jul 2017. (in Japanese).

7. The Ministry of Health, Labour and Welfare. http://www.mhlw. go.jp/bunya/kenkou/kekkaku-kansenshou28/pdf/kankoku_h25_ 6_01.pdf. 2017. Accessed 16 Jul 2017. (in Japanese).

8. The Ministry of Health, Labour and Welfare. http://www.mhlw. go.jp/file/05-Shingikai-10601000-DaijinkanboukouseikagakukaKouseikagakuka/0000161349.pdf. 2017. Accessed 16 Jul 2017. (in Japanese).

9. The Ministry of Health, Labour and Welfare. http://www.mhlw. go.jp/file/05-Shingikai-10601000-DaijinkanboukouseikagakukaKouseikagakuka/0000161329.pdf. 2017. Accessed 16 Jul 2017. (in Japanese).

10. Kinoshita T, Abe R, Hineno A, Tsunekawa K, Nakane S, Ikeda S. Peripheral sympathetic nerve dysfunction in adolescent Japanese girls following immunization with the human papillomavirus vaccine. Intern Med. 2014;53:2185-200.

11. Matsudaira T, Takahashi Y, Matsuda K, Ikeda H, Usui K, Obi T, et al. Cognitive dysfunction and regional cerebral blood flow changes in Japanese females after human papillomavirus vaccination. Neurol Clin Neurosci. 2016;4:220-7.

12. Anaya JM, Reyes B, Perdomo-Arciniegas AM, Camacho-Rodríguez B, Rojas-Villarraga A. Autoimmune/autoinflammatory syndrome induced by adjuvants (ASIA) after quadrivalent human papillomavirus vaccination in Colombian: a call for personalized medicine. Clin Exp Rheumatol. 2015;33:545-8.

13. Pellegrino P, Perrone V, Pozzi M, Carnovale C, Perrotta C, Clementi E, et al. The epidemiological profile of ASIA syndrome after HPV vaccination: an evaluation based on the vaccine adverse event reporting systems. Immunol Res. 2015;61:90-6.

14. Tanaka H, Fujita Y, Takenaka Y, Kajiwara S, Masutani S, Ishizaki Y, et al. Japanese clinical guidelines for juvenile orthostatic dysregulation version 1. Pediatr Int. 2009;51:169-79.

15. Low PA, Opfer-Gehrking TL, Textor SC, Benarroch EE, Shen WK, Schondorf R, et al. Postural tachycardia syndrome (POTS). Neurology. 1995;45:S19-25.

16. Okubo S. Digital plethysmogram. In: Kanai M, editor. Manual of clinical and laboratory medicine. 31st ed. Tokyo: Kanehara Publishing Inc.; 1998. p. 1546-7 (in Japanese).

17. Okazaki T, Saeki S, Hachisuka K. Adolescent normative data on simple neuropsychological tests for cognitive behavioral disorders: mini-mental State examination, trail making test, Wisconsin card sorting Test-KFS version and Miyake's verbal paired-associate learning test. Jpn J Rehabil Med. 2013;50:962-70 (in Japanese).

18. Nishioka K, Yokota S, Matsumoto Y. Clinical features and preliminary diagnostic criteria for human papillomavirus vaccination associated with neuroimmunopathic syndrome (HANS). Int J Rheum Dis. 2014;17:6.

19. Ushida T, Shibata M, Kitahara M, Yabuki S, Sumitani M, Murakami $\mathrm{T}$, et al. The effect of guidance regarding home exercise and ADL on adolescent females suffering from adverse effects 
after HPV vaccination in Japanese multidisciplinary pain centers. Pain Res Manag. 2016;2016:3689352.

20. Slade BA, Leidel L, Vellozzi C, Woo EJ, Hua W, Sutherland A, et al. Postlicensure safety surveillance for quadrivalent human papillomavirus recombinant vaccine. JAMA. 2009;302:750-7.

21. Bruehl S, Harden RN, Galer BS, Saltz S, Bertram M, Backonja $\mathrm{M}$, et al. Extensive validation of IASP diagnostic criteria for complex regional pain syndrome and proposed research diagnostic criteria. Pain. 1999;81:147-54.

22. Martínez-Lavín M. Hypothesis: human papillomavirus vaccination syndrome-small fiber neuropathy and dysautonomia could be its underlying pathogenesis. Clin Rheumatol. 2015;34:1165-9.

23. Carnovale C, Damavandi PT, Gentili M, Pozzi M, Antoniazzi S, Radice $\mathrm{S}$, et al. On the association between human papillomavirus vaccine and sleep disorders: evaluation based on vaccine adverse events reporting systems. J Neurol Sci. 2017;373:179-81.

24. Hirai T, Kuroiwa Y, Hayashi T, Uchiyama M, Nakamura I, Yokota $\mathrm{S}$, et al. Adverse effects of human papilloma virus vaccination on central nervous system: neuro-endocrinological disorders of hypothalamo-pituitary axis. Auton Nerv Syst. 2016;53:49-64.

25. Takahashi $Y$, Matsudaira $T$, Nakano $H$, Nasu $H$, Ikeda $H$, Nakaoka K, et al. Immunological studies of cerebrospinal fluid from patients with CNS symptoms after human papillomavirus vaccination. J Neuroimmunol. 2016;298:71-8.

26. Brinth L, Theibel AC, Pors K, Mehlsen J. Suspected side effects to the quadrivalent human papilloma vaccine. Dan Med J. 2015;62:A5064.

27. Brinth LS, Pors K, Theibel AC, Mehlsen J. Orthostatic intolerance and postural tachycardia syndrome as suspected adverse effects of vaccination against human papilloma virus. Vaccine. 2015;33:2602-5.

28. Palmieri B, Poddighe D, Vadalà M, Laurino C, Carnovale C, Clementi E. Severe somatoform and dysautonomic syndromes after HPV vaccination: case series and review of literature. Immuno Res. 2017;65(1):106-16.

29. Martínez-Lavín M. Fibromyalgia-like illness in 2 girls after human papillomavirus vaccination. J Clin Rheumatol. 2014;20:392-3.
30. Martínez-Lavín M, Martínez-Martínez LA, Reyes-Loyola P. HPV vaccination syndrome: a questionnaire-based study. Clin Rheumatol. 2015;34:1981-3.

31. Chandler RE, Juhlin K, Fransson J, Caster O, Edwards IR, Norén GN. Current safety concerns with human papillomavirus vaccine: a cluster analysis of reports in VigiBase $^{\circledR}$. Drug Saf. 2017;40:81-90.

32. Shoenfeld Y, Agmon-Levin N. ASIA-autoimmune/inflammatory syndrome induced by adjuvants. J Autoimmun. 2011;36:4-8.

33. Perricone C, Colafrancesco S, Mazor RD, Soriano A, AgmonLevin N, Shoenfeld Y. Autoimmune/inflammatory syndrome induced by adjuvants (ASIA): unveiling the pathogenic, clinical and diagnostic aspects. J Autoimmun. 2013;47:1-16.

34. Hendrickson JE, Tormey CA. Human papilloma virus vaccination and dysautonomia: considerations for autoantibody testing and HLA typing. Vaccine. 2016;34:4468.

35. Blitshteyn S, Brook J. Postural tachycardia syndrome (POTS) with anti-NMDA receptor antibodies after human papillomavirus vaccination. Immunol Res. 2017;65(1):282-4.

36. Gisselquist DP. Estimating HIV-1 transmission efficiency through unsafe medical injections. Int $\mathrm{J}$ STD AIDS. 2002;13:152-9.

37. Mahboobi N, Agha-Hosseini F, Safari S, Lavanchy D, Alavian SM. Hepatitis B virus infection in dentistry: a forgotten topic. J Viral Hepat. 2010;17:307-16.

38. Wilder RT. Management of pediatric patients with complex regional pain syndrome. Clin J Pain. 2006;22:443-8.

39. The Ministry of Health, Labour and Welfare. http://www.mhlw. go.jp/file/05-Shingikai-10601000-DaijinkanboukouseikagakukaKouseikagakuka/0000161329.pdf. 2017. Accessed 16 Jul 2017. (in Japanese).

40. Aratani S, Fujita H, Kuroiwa Y, Usui C, Yokota S, Nakamura I, et al. Murine hypothalamic destruction with vascular cell apoptosis subsequent to combined administration of human papilloma virus vaccine and pertussis toxin. Sci Rep. 2016;6:36943. 